n'en est pas moins un progrès important, car avant que les mieroorganismes qui rendent le beurre " aigre" n'aient disparu, on ne remarque pas du tout que le beurre est aussi disposé à devenir " huileux ", et on ne peut pas, par conséquent, auparavant découvrir et corriger aussi ce défaut.

Si on doit corriger un défaut, il faut d'abord savoir auquel on a affaire, et c'est ici qu'à notre avis l'épreuve de la catalase peut être utilisée avec succès.

Nous maintenons, à nouveau, que les principaux résultats des recherches susindiquées sont les suivants:

$1^{\circ}$ L'indice de catalase donne une expression remarquable de la teneur du beurre en microorganismes étrangers et se détermine d'une façon très simple.

$2^{\circ}$ Pour un certain nombre de défauts du beurre, au fur et à mesure que le défaut se développe, a lieu une augmentation correspondante, mais un peu antérieure, de l'indice de catalase, ce qui prouve que les défauts en question et la catalase ont la même origine, c'est-à-dire les microorganismes étrangers. Nous appelons ces défauts défauts "bactériens".

$3^{\circ}$ Pour un autre groupe de défauts du beurre, il n'y a aucun rapport avec l'indice de catalase, et le beurre devient mauvais sans contenir de catalase. Ces défauts sont dus, soit à des microorganismes qui ne forment pas de catalase, soit, ce qui est certainement plus fréquent, à des modifications purement chimiques, et nous les appelons défauts "chimiques".

$4^{\circ} \mathrm{Le}$ bon beurre qui se conserve bien a presque toujours un indice de catalase bas.

$5^{\circ}$ Nous avons toujours trouvé l'indice de catalase 0 pour le beurre tout à fait frais.

(A suivre.)

\title{
LE LIQUIDE DANS LE BEURRE (1)
}

\section{par \\ B. VAN DER BURG}

Professeur à l'Ecole supérieure d'Agriculture de Wageningen (Pays-Bas). Conférence d'ouverture de la Journée de laiterie à Wageningen, le 30 juin 1932.

Comme titre de la Conférence d'ouverture de la première Journée de laiterie de la Semaine agricole néerlandaise, j'ai choisi le suivant : Le liquide dans le beurre.

Lorsque nous faisons le relevé de toutes les questions que nous

(1) Extrait de Het Nederlandsch Weekblad voor Zuivelbereiding en-Handel, $\mathrm{n}^{0} 14,5$ juillet 1932. 
pouvons classer sous cette rubrique, et que nous pourrions done examiner, nous nous apercevons bien vite que l'ensemble de ces questions constitue un terrain tellement vaste, que pour être complets nous devrions passer en revue toute la préparation du beurre, depuis les manipulations de la crème jusqu'à l'achèvement et l'emballage, et même la conservation du produit.

Il est donc bien évident que j'ai dû me limiter beaucoup, et me borner à n'examiner maintenant que quelques aspects de ce vaste sujet. Je devrai passer sous silence nombre de questions, qui pourraient être examinées dans une autre conférence, laquelle aurait le même titre que celui que j'ai choisi aujourd'hui.

De toute évidence, il est intéressant pour nous de connaître la quantité d'eau qui se trouve dans le beurre, dans les conditions normales ; mais pas moins importante que la question de la teneur en eau du beurre, est celle de la composition du liquide que contient le beurre, et de plus, nous ne pouvons nous désintéresser de la répartition du liquide dans le beurre. Ces questions en soulèvent encore d'autres ; par exemple :

Quelles sont les conditions qui déterminent la teneur en eau du beurre, et peut-on les modifier à volonté lors de la préparation du produit?

Quels sont les facteurs qui prédominent dans la composition du liquide dans le beurre?

Quelle est l'influence de la composition de ce liquide sur l'arome, sur la saveur et surtout sur la faculté de conservation du beurre?

Quelle est l'influence du mode de répartition de ce liquide sur les propriétés, par exemple sur l'aspect du beurre?

Et enfin :

Que pouvons-nous faire pour obtenir les conditions les plus favorables concernant la quantité, la composition et la répartition du liquide dans le beurre?

LE Pourcentage d'eAu du BeuRre. - En premier lieu notre attention va se fixer pendant quelques instants sur le pourcentage d'eau du beurre.

Avant l'organisation du contrôle des beurres dans notre pays, le "beurrier" se préoccupait peu de la quantité d'eau que pouvait contenir le beurre. Telle qu'elle était déterminée par la préparation, elle lui paraissait satisfaisante. Il savait que la teneur en eau d'un beurre normal oscillait entre 11,12 et $15 \%$ environ, et que le beurre salé contenait approximativement un demi pour cent de moins d'eau que le beurre non salé. Le beurrier d'antan, en général, n'essayait pas d'élever à volonté la teneur en eau du beurre.

Cependant, l'art d'incorporer un surplus d'eau dans le beurre 
n'était pas inconnu dans le pays, mais il était moins pratiqué par ceux qui préparaient le beurre que par certains marchands et mélangeurs de beurre qui, par leurs pratiques, ont à cette époque causé un préjudice considérable à la réputation du beurre néerlandais.

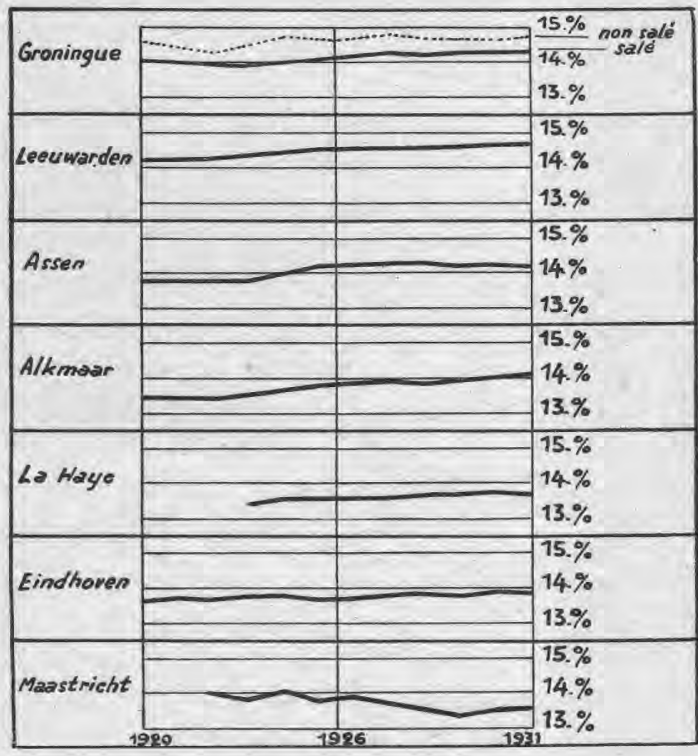

Fig. 1.

Teneur moyenne en eau du beurre contrôlé de 1920 à 1931 selon des données des rapports annuels des Stations de contrôle des beurres.

Notre loi sur le beurre ne mentionne pas de limite maximum autorisée de la teneur en eau du beurre ; mais par la détermination $d$ 'une teneur en matière grasse minimum de $80 \%$, une limite maximum de la teneur en eau est pré. cisée, évidemment d'une manière indirecte.

Pour les affiliés aux Stations néerlandaises de contrôle du beurre, depuis la création de ces organismes, tant pour le beurre non salé que pour le beurre salé, la teneur en eau maximum a été fixée à $16 \%$. Vers 1911, les Stations de contrôle adoptèrent un maximum de $15 \frac{1}{2} \%$, qui, lorsqu'il est dépassé, rend l'affilié passible d'amende.

Les données fournies par les rapports annuels des Stations de contrôle du beurre nous permettent de nous représenter assez exactement quelle est la moyenne de la teneur en eau du beurre néerlandais.

Nous servant des données de ces rapports de 1920 à 1931, pour autant qu'ils étaient eomplets, nous avons élaboré des tracés (v. Fig. 1).

Ces tracés nous montrent clairement, pour autant que les données concernant le beurre salé et le beurre non salé sont fournies séparément (Groningue), que la moyenne de la teneur en eau du premier est inférieure d'environ $1 / 2$ p. 100 à celle du second.

De plus, il apparaît que la teneur en eau du beurre de la Frise (Leeuwarden) est toujours plus élevée que celle du beurre du Limbourg (Maastricht) et également que celle du beurre du Brabant 
(Eindhoven). Le fait que les échantillons examinés à Maastricht sont prélevés pour ainsi dire exclusivement sur des beurres non salés, - ce qui certainement n'est pas le cas pour Leeuwarden, - ne pouvait nous faire prévoir l'écart de la teneur en eau des échantillons de beurre de ces deux stations, dans cette direction.

Si nous cherchons une explication du phénomène constaté, nous nous demandons immédiatement si la circonstance que le beurre du Limbourg est en général un peu plus consistant que celui de la Frise ne pourrait pas être cause d'une élimination moins prononcée d'eau lors du malaxage de ce dernier, d'où sa teneur en eau plus élevée. La réponse à cette question doit être: Probablement pas; et ce n'est pas là que nous devons chercher la cause de l'écart. En fait, le beurre danois, dont généralement la consistance est meilleure que celle de notre beurre, présente en moyenne une teneur en eau plus élevée que le beurre néerlandais; l'écart n'en est pas moins de 0,30 à $0,40 \%$.

D'ailleurs, les tracés montrent que dans certaines régions du pays la teneur en eau a subi progressivement une légère hausse au cours de ces années. La teneur en eau du beurre de la Frise tend à s'élever vers $15 \%$. Je ne trouve d'autre explication de ce fait, si ce

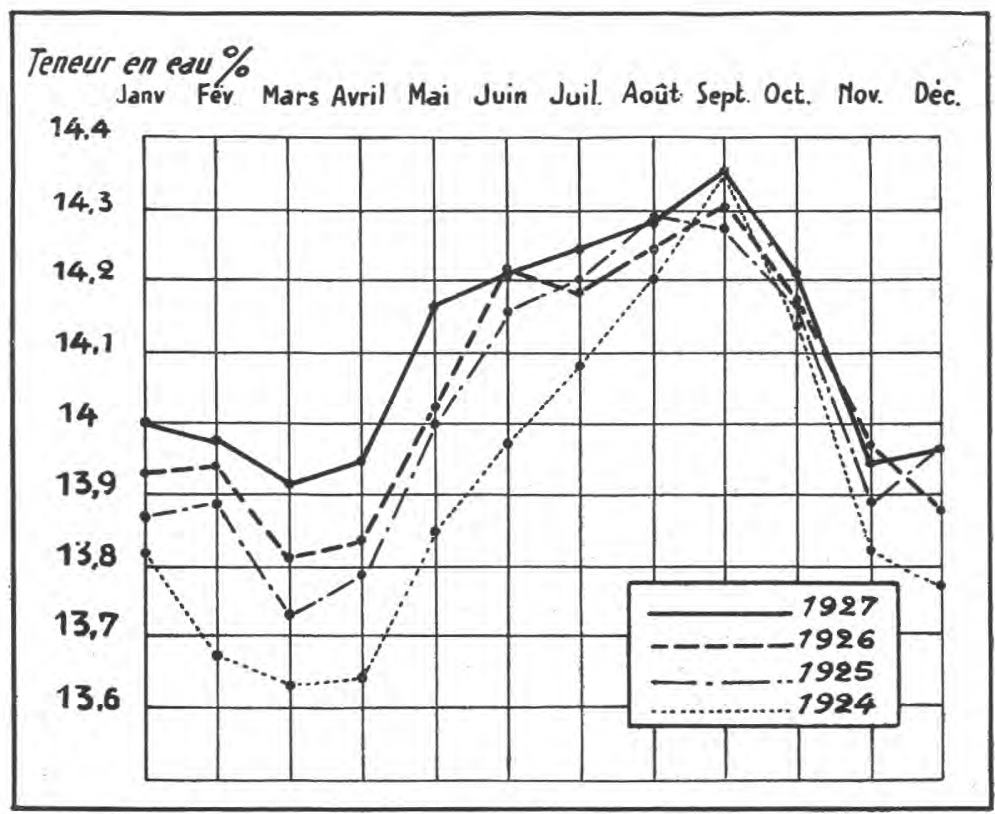

Fig. 2.

Teneur moyenne en eau du beurre néerlandais au cours des différents mois de l'année. 

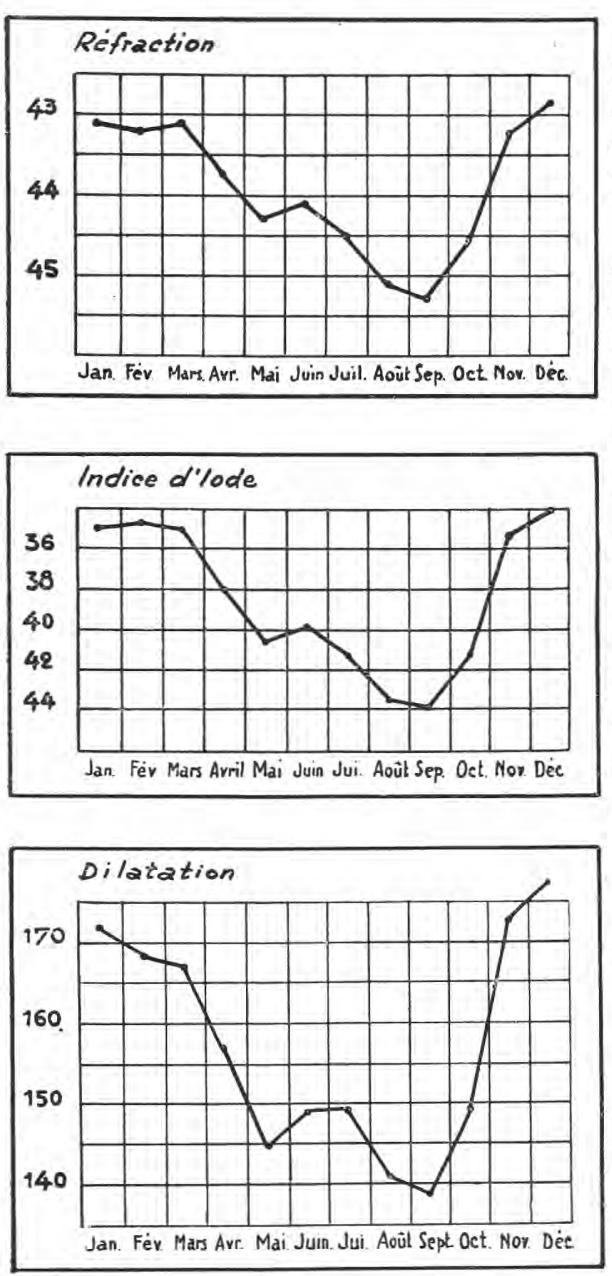

Fig. 3. Rapport entre les propriétés physiques et
chimiques de la matière grasse du beurre (réfraction et indice d'iode) et la dilatation de la crème, d'après les données des recherches de van Dam et Sirks [3]. Pour la détermination de la dilatation, la teneur en matière grasse de la crème avait été réglée à $45 \%$; la crème avait été maintenue pendant 21 heures à $12^{\circ} \mathrm{C}$.; la détermination des dilatations fut faite pour des températures allant de 13 à $28^{\circ} \mathrm{C}$, et ces dilatations furent exprimées en $\frac{1}{10.000}$. n'est la tentative d'amener, par des procédés techniques, la teneur en eau du beurre aussi près que possible de la limite maximum autorisée.

Cependant la technique ne constitue pas le seul facteur déterminant la teneur en eau du beurre. Ce fait est nettement démontré par le tracéque j'ai emprunté à une publication de F. Kenstra [1]. Ce tracé montre que d'année en année la teneur en eau du beurre néerlandais atteint un sommet au mois de septembre (Fig. 2). Antérieurement déjà, MEYERINGH [2] fit la même observation. Ce phénomène est évidemment en corrélation avec les propriétés physiques de la matière grasse du beurre, qui, elles, dépendent naturellement de sa composition chimique.

Des recherches de VAN DAM et Sirks [3] démontrèrent que la composition de la matière grasse du lait est fréquemment telle vers la fin de l'été dans notre pays, que, même en ayant recours aux procédés techniques les plus perfectionnés, on ne parvient pas à en préparer du beurre ayant une bonne consistance.Telfutle cas pour le tiers environ des échantillons de crème examinés au cours de toute la période du séjour des vaches en prairie.

J'ai construit des tracés 
en me basant sur les chiffres fournis par l'enquête approfondie de van Dam et Sirks (Fig. 3).

Pour l'élaboration de ce tracé on utilisa les données d'examens de plus de 500 échantillons de crème fournis par une vingtaine d'usines de laiterie de toutes les régions du pays. Nous voyons que la dilatation de la matière grasse de la crème (après refroidissement de tous les échantillons dans les mêmes conditions) est le moins prononcée vers la fin de l'été. Cette dilatation est une mesure objective de la tendance à la cristallisation, et par là même, de la consistance de la matière grasse. La corrélation de la dilatation avec la réfraction et l'indice d'iode, pour lesquels les ordonnées sont négatives dans cette figure, - ce qui donne plus de clarté, - apparaît immédiatement ici.

Nous déduisons de ce qui précède, que la teneur en eau du beurre présente une tendance à s'élever nettement caractérisée durant la période au cours de laquelle, à la suite de certaines propriétés spéciales de la matière grasse du beurre, nous obtenons fréquemment du beurre à consistance molle.

Ayant ainsi conclu, j'interromps mon exposé sur le pourcentage d'eau du beurre, pour examiner plus attentivement la composition du liquide dans le beurre et le mode suivant lequel il s'y présente.

LA composition du liquide du beurre. - Pour cet examen, nous n'allons pas partir des données d'analyse qui nous sont connues à ce sujet, mais nous allons essayer d'en obtenir la compréhension, en examinant ce qui nous est connu de la formation du beurre par le barattage de la crème, et nous déduirons de ces faits quelle est la composition du liquide. Nous ne pouvons le faire sans un exposé de quelques aspects de la théorie du processus du barattage.

Lorsque nous comprimons, en une mince couche, entre deux lamelles de verre, un petit granule de beurre bien travaillé, et que nous examinons cette préparation, au microscope, à un grossissement approprié, nous constatons que dans le champ microscopique se trouvent de nombreux espaces clairs, à contours plus ou moins circulaires. En examinant de plus près, nous nous rendons compte que ces espaces clairs sont les images d'objets plus ou moins sphériques, constitués par une substance qui réfracte moins la lumière que le milieu dans lequel ils se trouvent; et la conclusion s'impose, que nous avons affaire à des gouttelettes de liquide qui sont dispersées dans la matière grasse (Fig. 4).

La présence du liquide dans le beurre en très fines gouttelettes a donné lieu à l'élaboration d'une hypothèse concernant la formation du beurre au cours du barattage. En fait, l'image microscopique fait supposer que le beurre est une émulsion du type eau dans huile; 
ici, donc, des gouttelettes du liquide dans la matière grasse du beurre. Le fait que la matière grasse du beurre n'est pas liquide, et que le milieu de dispersion est, par conséquent, solide, ou du moins semisolide, est dans ce cas d'importance secondaire. Certains auteurs,

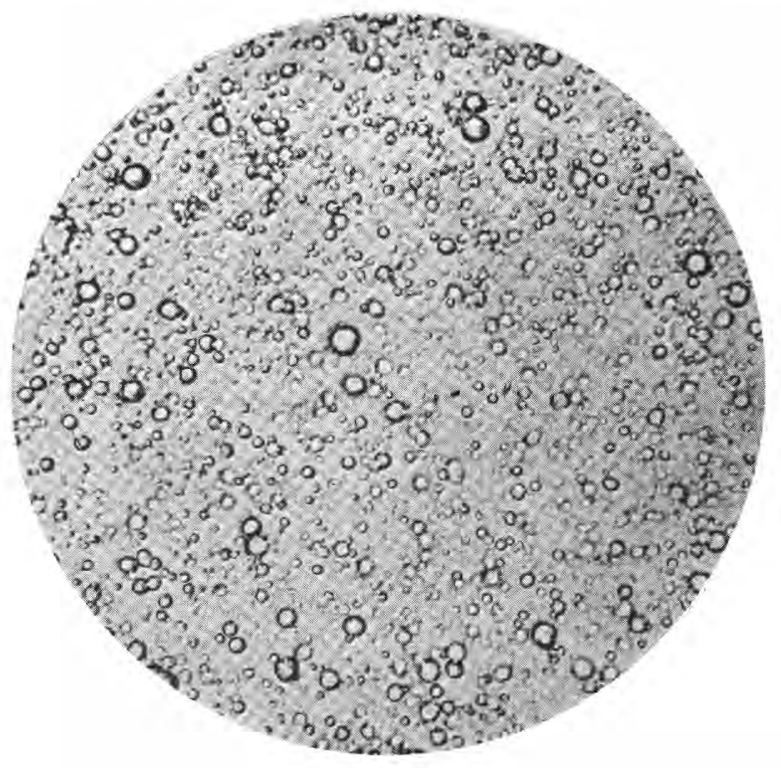

Fig. 4.

Gouttelettes de liquide dans du beurre achevé.

Grossissement : $400 \mathrm{X}$.

comme, par exemple, Hunziker [4], croient pouvoir expliquer le processus du barattage en admettant qu'il se produit une inversion du type d'émulsion lors du barattage, que probablement l'émulsion primitive de matière grasse du lait dans le sérum de lait se transforme en émulsion de sérum de lait dans la matière grasse du beurre.

L'inversion des émulsions est un phénomène bien connu. Il est facile de transformer une émulsion d'huile d'olive dans une solution très diluée de soude en une émulsion d'eau dans de l'huile par l'addition d'une petite quantité de chlorure de calcium à l'émulsion primitive. A la question de savoir si telle inversion est possible uniquement sous l'influence de facteurs mécaniques, comme cela devrait se produire lors du barattage de la crème, je devrais répondre que cette possibilité ne doit pas être théoriquement exclue, bien que, dans les publications qu'il m'ait été donné de consulter, aucun exemple n'en est mentionné, en dehors du processus du barattage, 
pour lequel, selon certains auteurs, telle inversion se produirait. Ici cependant, rien ne peut m'en convaincre.

Au lieu d'utiliser du beurre achevé pour notre préparation, nous prendrons un granule de beurre qui n'a pas été malaxé. Comme

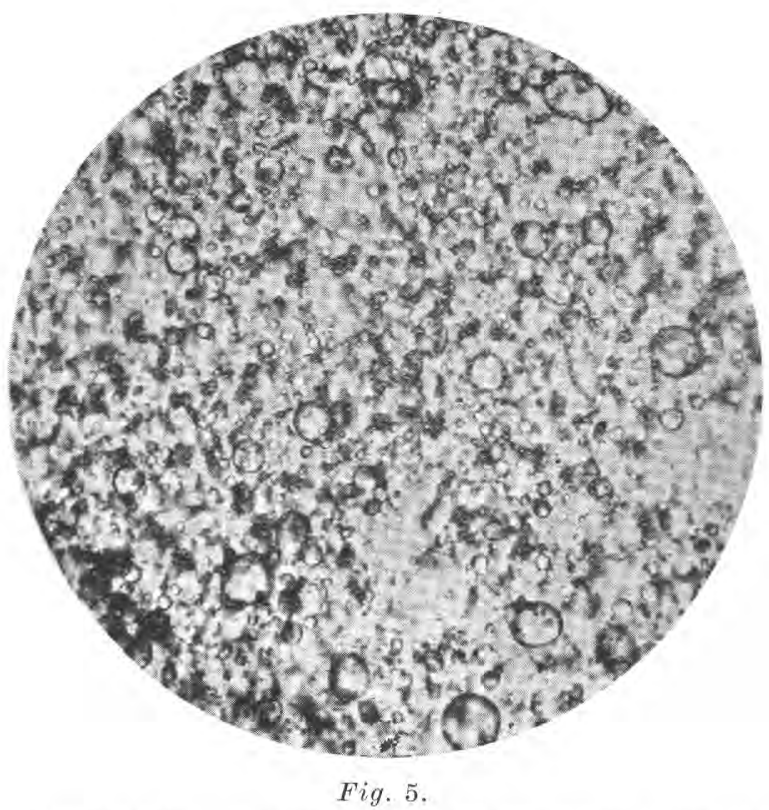

Gouttelettes de liquide dans du beurre non travaillé. Grossissement : $400 \mathrm{X}$.

nous devrons comprimer ce granule entre les deux lamelles de verre pour obtenir une préparation transparente, un léger malaxage va se produire. En présence de l'impossibilité de l'éliminer, nous le réduirons au minimum, en prenant un granule de très petite dimension, n'exigeant qu'une très légère compression, dans l'espoir d'éviter une modification trop prononcée. C'est ce que montre la microphotographie de cette préparation (Fig. 5).

L'image obtenue est complètement différente de celle du beurre achevé. Les gouttelettes de liquide ont des dimensions bien plus considérables et des contours bien plus irréguliers. Dans le beurre malaxé, les globules de matière grasse présentent une déformation et une compression bien plus prononcées et les gouttelettes de liquide, primitivement de dimension relativement considérable, ont été fractionnées en gouttelettes plus petites; d'où il résulte que, par le malaxage, le beurre a acquis les caractéristiques d'une émulsion de liquide dans la matière grasse. Cependant, ceci ne rend pas pro- 
bable que le nouveau type d'émulsion est produit par le barattage. Certaines exigences techniques rendent, en effet, relativement peu probable la production d'une inversion des conditions d'émulsion par le processus du barattage. En fait, si nous voulons obtenir le maximum de perfection qu'il est possible de réaliser pour le processus. du barattage, nous prendrons soin lorsque le barattage débute, que la matière grasse de la crème soit solide et en équilibre avec la température de barattage. Il me paraît des plus inadmissibles que cette matière grasse, en équilibre en ce qui concerne ses propriétés physiques, son état, solide ou semi-solide, puisse confluer en une phase continue, dans laquelle du sérum de lait serait réparti comme phase dispersée.

Je suis d'avis qu'en eux-mêmes, les faits que je viens d'exposer permettent de rejeter la théorie du barattage qui admet que la formation du beurre au cours de celui-ci est le résultat d'une inversion du type d'émulsion. Plus loin, nous verrons que l'on peut encore avancer d'autres faits venant infirmer cette théorie.

$$
\text { * * * }
$$

La théorie la plus récente du barattage est celle dite de l'écume de RAHN. Cet auteur établit un rapport entre la formation d'écume qui se produit lors du barattage de la crème et la formation du beurre [5].

C'est un fait bien connu, qu'il se produit, lors du barattage, une écume abondante. L'on sait également que la formation d'écume s'arrête, au début de la formation des granules de beurre visibles, lorsque se produit la désagrégation de la erème. A ce sujet, il est intéressant de rappeler qu'on construit des barattes dans lesquelles la formation de beurre est amenée par le passage d'air à travers la crème, à la suite duquel il se forme une écume abondante. Je rappelle encore ici les expériences de SiEdel [6], déjà vieilles d'une trentaine d'années, où il fut démontré que, même après des heures de barattage, il ne se formait aucun beurre dans de la crème qui avait été traitée de façon à empêcher la production d'écume.

Quelle est maintenant la conception de RAHN en ce qui concerne le rapport entre la formation d'écume dans la crème et la formation du beurre? En résumé, la voici :

Les forces qui préviennent la confluence des globules de matière grasse dans le lait ou dans la crème, qui constituent donc la cause de la stabilité de l'émulsion, sont des forces qui s'exercent à la surface de séparation des milieux. Nous ne pouvons apporter des modifications par l'addition de produits chimiques ; cette addition nous mettrait en conflit avec les déterminations légales concernant les produits alimentaires. Nous cherchons d'autres moyens; en fait, 
nous allons créer de nouvelles surfaces limitantes. Nous les obtenons en incorporant de l'air dans la masse, en provoquant donc une formation d'écume dans la crème. En procédant ainsi, nous obtenons une modification complète du système.

La substance qui, selon RAHN, rend possible la formation d'écume dans la crème, est la même que celle qui se trouve dans la surface limitante entre la matière grasse et le sérum de lait ; elle cause une diminution de la tension superficielle et prévient la confluence des globules de matière grasse. Il n'est pas possible de déterminer exactement quelle est cette substance ; il est probable que c'est une protéine. Comme, à mon avis, une connaissance exacte de sa nature n'est pas indispensable ici, je m'autoriserai, avec RAHN, à ne lui donner d'autre nom que celui de «substance de l'écume ».

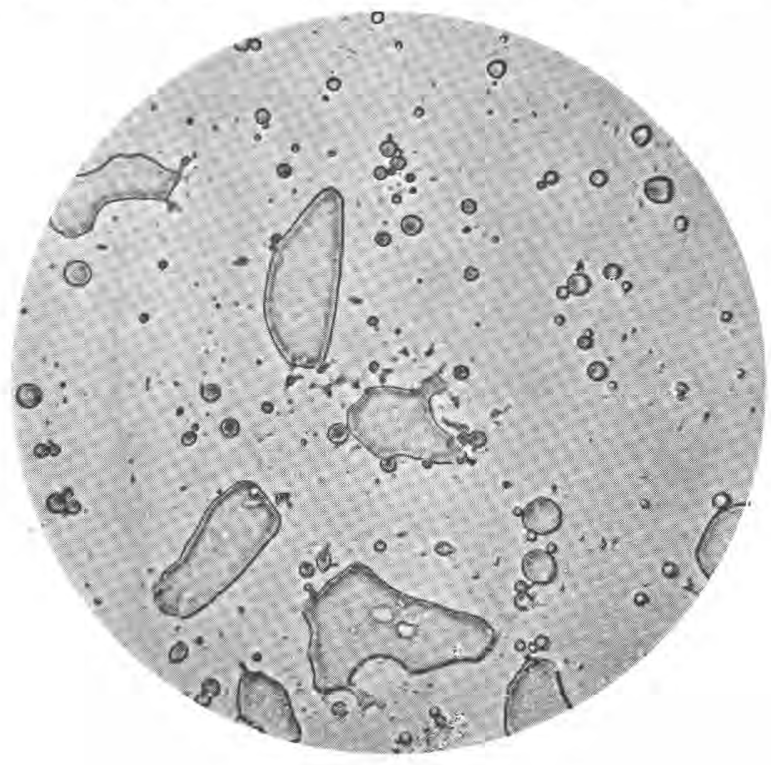

Fig. 6.

Granules de beurre formés peu de temps après le début du barattage. Grossissement : $400 \mathrm{X}$.

Cette substance de l'écume, donc, s'amasse en les lamelles d'écume, et selon la conception du processus du barattage de RAHN, entraîne les globules de matière grasse, qui s'amassent donc dans les parois des bulles d'air de l'écume. Ces parois sont constituées par deux lamelles entre lesquelles se trouve un liquide qui n'est autre que du sérum de lait. Le liquide s'écoule, abandonnant, dans les parois, des bulles d'air qui s'amincissent par l'évacuation du sérum de lait, les globules de matière grasse, lesquels, comprimés les uns contre les 
autres, se soudent en granules minuscules. Au contact de l'air qui se trouve des deux côtés des parois des bulles d'air, la substance de l'écume se solidifie, devient friable; enfin, les lamelles de l'écume se brisent, mettant en liberté les conglomérats de globules de matière grasse. Par le travail mécanique, ces conglomérats de beurre, invisibles à l'œil nu, sont réunis en amas plus grands ; puis des granules visibles se forment et la consistance de la crème se modifie. La

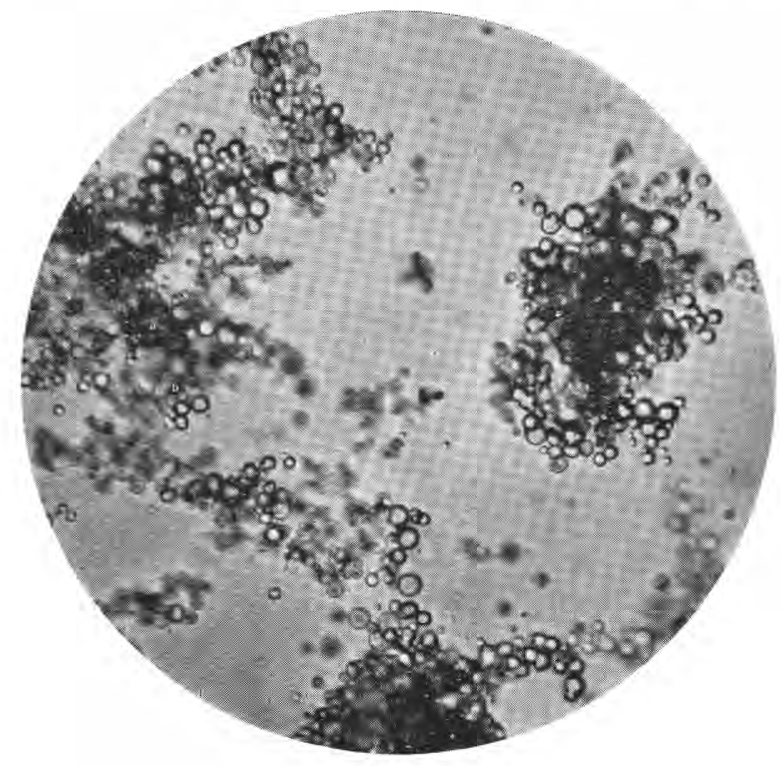

Fig. 7.

Agglutination (formation de flocons) de globules de matière grasse. Grossissement : $400 \mathrm{X}$.

crème se désagrège; le volume des granules de beurre augmente, et généralement à un degré tel que bientôt le barattage est terminé, La fin du barattage est atteinte dès qu'on parvient à séparer facilement les granules de beurre formés et le babeurre.

La microphotographie (Fig. 6) montre le mode de conglomération des globules de matière grasse en granules de beurre. Je la compare avec une photographie de globules de matière grasse conglomérés en flocons sans qu'il y ait eu barattage. Cette photographie (Fig. 7) montre ce qui se produit dans du lait qui a été laissé quelque temps au repos, et surtout lorsque celui-ci a été préalablement refroidi à basse température. Ce phénomène est appelé agglutination ou formation de flocons. Ainsi que van Dam [7] et Rahn [8] l'ont démontré, indépendamment l'un de l'autre, et pour ainsi dire en 
même temps, cette agglutination est indispensable pour une montée rapide de la crème (1).

Lorsque nous comparons les deux photographies, nous voyons immédiatement que les globules de matière grasse sont bien moins serrés les uns contre les autres dans les flocons résultant de l'agglutination que dans les granules de beurre résultant du barattage. Une simple expérience nous démontrera que nous nous trouvons devant deux phénomènes différents. Nous n'avons qu'à chauffer le matériel des préparations au delà du point de fusion de la matière grasse; la température de $40^{\circ} \mathrm{C}$. est suffisante. Les flocons, formés par l'agglutination, se désagrègent par séparation des globules de matière grasse; nous obtenons une goutte de matière grasse au lieu d'un granule de beurre à la suite de la confluence des globules de matière grasse qui constituaient ce granule. Dans l'agglutination, nous nous trouvons devant un processus réversible, tandis que le processus par lequel se forme le beurre est irréversible.

La théorie émise par RAHN en ce qui concerne le processus de barattage, doit être considérée, en fait, comme une extension de la théorie, jusqu'ici toujours en honneur, entre autres dans les manuels néerlandais traitant de la préparation du beurre, qui explique la formation du beurre en admettant que par l'agitation de la crème les globules de matière grasse sont projetés les uns contre les autres de façon à former des conglomérats. D'ailleurs, telle est l'explication fournie par HUNZIKER [9] du début de la formation du beurre jusqu'à la désagrégation de la crème. D'a près cet auteur, l'inversion de l'émulsion se produit au moment où s'arrête la formation de l'écume.

Il est incontestable que la théorie de RAHN est très attrayante et permet l'explication de phénomènes qui, autrement, resteraient inexpliqués. Cependant, elle ne nous fournit pas de solution pour certains problèmes, laissant ouvert ainsi un champ de recherches ultérieures. En ce moment cependant, il n'y a pas lieu d'insister à ce sujet.

(A suivre.)

\section{MAIVIVITE COLIBACILLAIRE PARAPLÉGIQUE DE LA VACHE (2)} par MM. LESBOUYRIES, ADAM et ARGOUD.

L'étude systématique que nous avons entreprise sur l'étiologie des mammites, nous a conduits à nous occuper d'une forme de mammite s'accompagnant de paraplégie.

(1) B. van Der Burg. Étude du lait considéré comme émulsion. Le processus de la montée de la crème. Le Lait, p. 452 à 466 (1927).

(2) Extrait du Bulletin de l'Acalémie vétérinaire de Francs, 1932, n $\mathrm{n}^{\circ} 7, \mathrm{p} .321$. 